https://doi.org/10.15407/ujpe66.4.310

V.I. MASLOV ${ }^{1,2}$ O.K. CHEREMNYKH, ${ }^{1}$ A.P. FOMINA, ${ }^{3}$ R.I. KHOLODOV ${ }^{4}$ O.P. NOVAK, ${ }^{4}$ R.T. OVSIANNIKOV ${ }^{5}$

${ }^{1}$ Space Research Institute, Nat. Acad. of Sci. of Ukraine and the State Space Agency of Ukraine (40, 4/1, Glushkov Ave., Kyiv 03680, Ukraine)

2 NSC Kharkiv Institute of Physics and Technology, Nat. Acad. of Sci. of Ukraine (1, Academichna Str., Kharkiv 61108, Ukraine; e-mail: vmaslov@kipt.kharkov.ua)

${ }^{3}$ Bogolyubov Institute for Theoretical Physics, Nat. Acad. of Sci. of Ukraine (14b, Metrolohichna Str., Kyiv 03143, Ukraine)

${ }^{4}$ Institute of Applied Physics, Nat. Acad. of Sci. of Ukraine (58, Petropavlivska Str., Sumy 40000, Ukraine)

${ }^{5}$ Karazin Kharkiv National University (4, Svobody Sq., Kharkiv 61022, Ukraine)

\title{
VORTEX STRUCTURES AND ELECTRON BEAM DYNAMICS IN MAGNETIZED PLASMA
}

We investigate the formation of vortex structures at the reflection of an electron beam from the double layer of the Jupiter ionosphere. The influence of these vortex structures on the formation of dense upward electron fluxes accelerated by the double layer potential along the Io flux tube is studied. The phase transition to the cyclotron superradiance mode becomes possible for these electrons. The conditions of the formation of vortex perturbations are considered. The nonlinear equation that describes the vortex dynamics of electrons is constructed, and its consequences are studied.

Keywords: electron beam dynamics, double electric layer, mechanism of electron reflection, Jovian ionosphere, plasma, vortices.

\section{Introduction}

In this paper, the vortex dynamics of electrons is considered, and the processes that can influence the formation of upward electron fluxes in plasma double layers (DLs) are studied in the framework of the cyclotron superradiance (CSR) effect. The possibility of the formation of vortex structures due to the interaction of electron currents with the Jupiter ionosphere plasma is discussed.

The influence of Io on Jupiter's magnetosphere and its radio emission has been studied for a long time $[1,2]$. This active satellite is constantly erupting particles into the ionosphere and is connected with the generation of the strong radio emission from Jupiter which was discovered in 1955. The interaction between Io and Jupiter occurs in the form of electric currents that moves from Io along the Jupiter magnetic field lines and through the ionosphere near the planet poles, by forming the Io magnetic flux tube

(C) V.I. MASLOV, O.K. CHEREMNYKH, A.P. FOMINA, R.I. KHOLODOV, O.P. NOVAK, R.T. OVSIANNIKOV, 2021
(IFT). Near the footprint of the IFT, where the particles carrying this current influence the Jupiter atmosphere, auroras arise. They are observed in the form of bright tails of radiation stretched behind the main spot along the movement of the IFT footprint on the planet surface [3, 4]. In addition, the projections of other satellites are also visible, but with much lower brightness. The radiation spot of Io at a certain point has the shape of ellipse with a size $\sim 200-500 \mathrm{~km}$, which is slightly larger than the projection size of the satellite itself onto the planet near $\sim 200 \mathrm{~km}$ (due to the IFT constriction). It indicates that the Io-Jupiter interaction region is slightly larger than the size of Io. The IFT tail is less bright in the northern hemisphere, where the magnetic field is stronger than in the southern hemisphere. Conversely, the radio emission is more intense in northern areas [5,6]. Jupiter's equatorial field strength is 4.3 Gauss, ranging from 10 Gauss at the south pole to 14 Gauss at the north pole.

Since 2016, NASA's Juno spacecraft is orbiting aroud Jupiter. It is equipped with devices to compile the detailed information about the planet. Based on these data, new effects were discovered, for ex-

ISSN 2071-0194. Ukr. J. Phys. 2021. Vol. 66, No. 4 
ample, the observations report on the distinct, highenergy, discrete electron acceleration in Jupiter's auroral polar regions and on upward magnetic-fieldaligned electric potentials of up to $400 \mathrm{keV}$, by one order of magnitude larger than the largest potentials observed at Earth. Juno's Energetic particle Detector Instrument (JEDI) detected intense electron beams moving away from Jupiter's polar regions. In those beams, there are often found electrons with energies above $\sim 1 \mathrm{MeV}$, sometimes up to $>10 \mathrm{MeV}$. These beams occur primarily above the swirl region of the polar cap aurora. The correlation between the swirl emergence from an Ultraviolet Spectrograph (UVS) and the very intense beams from JEDI was found in $[4,7,8]$. We note that, in literature, some additional mysteries concerning the precise nature of this acceleration still exist.

In works [10-14], the original model was proposed that describes the generation of a super-powerful Jovian radio emission, which is based on the effect of the Fomin-Dicke collective coherent CSR for a system of inverted electrons at high Landau levels in rarefied magnetized plasma. According to the model, electron beams are accelerated from Io toward Jupiter and are reflected from double electric layers that arise in the plasma of the ionosphere. Then the reflected electron beams move upward and pass into the coherent superradiance CSR mode. The criterion of the phase transition to the CSR mode depends, in particular, on such characteristics of electron beams as the density and temperature.

The DL phenomenon has been studied for a long time (see [15-21]) and was observed under laboratory conditions and in astrophysical applications. In addition, DLs are observed in auroral regions, where they require some external drivers to induce the electron acceleration $[22,23]$. According to observations, Jupiter's auroras sometimes have parallel potential DLs analogously to Earth's aurora. The electric DLs are plasma regions with a violated quasineutrality and with a size of several Debye radii. The formation of such layers is possible at the border between regions in plasma with different characteristics, for example, with different temperatures. The formation mechanisms of sufficiently strong DLs are also possible, associated with the injection of particles into the plasma, due to the current amplification of the plasma instability. Most of the beam electrons are reflected in the ionosphere by parallel electrical layers

ISSN 2071-0194. Ukr. J. Phys. 2021. Vol. 66, No. 4

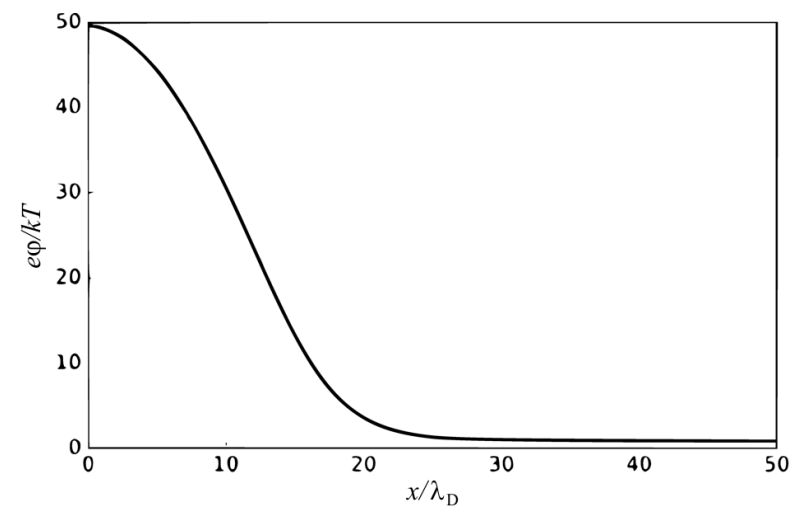

Fig. 1. Potential profile of the double layer

and cause auroras, although some of the electrons are scattered.

Numerical simulations in [14] showed the possibility of a quasi-stationary DL formation during the interaction of an electron beam with a plasma in the nonrelativistic case. When the electron beam with a density of $10^{4} \mathrm{~cm}^{-3}$ is injected into the plasma of the Jupiter ionosphere, a DL can be formed in the region, where the densities of the beam and plasma are approximately equal. The phase portrait of DL and the distribution of free and trapped components of the electron beam were found. It also shows the formation of cold electron beams, for which a transition to the CSR mode is possible. A characteristic potential drop is observed in the DL region. The DL potential is shown in Fig. 1.

The presented distribution was obtained by averaging the values of potential and field strength over a time interval exceeding much the period of plasma oscillations. The instantaneous values can be significantly distorted due to plasma oscillations. As seen in Fig. 1, the DL has a width of approximately $20 r_{d e}$. The potential drop in the DL is determined by the energy of the electron beam. For example, for the following parameters of plasma electrons - concentration $n_{0} \approx 10^{4} \mathrm{~cm}^{-3}$ and temperature $k T \approx 1 \mathrm{keV}$, we get the Debye radius $r_{d e} \approx 2.4 \mathrm{~m}$, plasma frequency $\omega_{p}^{-1} \approx 1.8 \times 10^{-7} \mathrm{~s}$ and, accordingly, the width of DL is $\sim 50 \mathrm{~m}$, the time of formation $\sim 10^{-6} \mathrm{~s}$. In this case, the energy of the beam is $50 \mathrm{keV}$, which corresponds to the DL potential of $50 \mathrm{kV}$.

The numerical simulation of a double layer was carried out in the region of $100 r_{d e}$ with open boundary conditions. At the initial moment of time, it was assumed that the region is filled with an equilibrium 


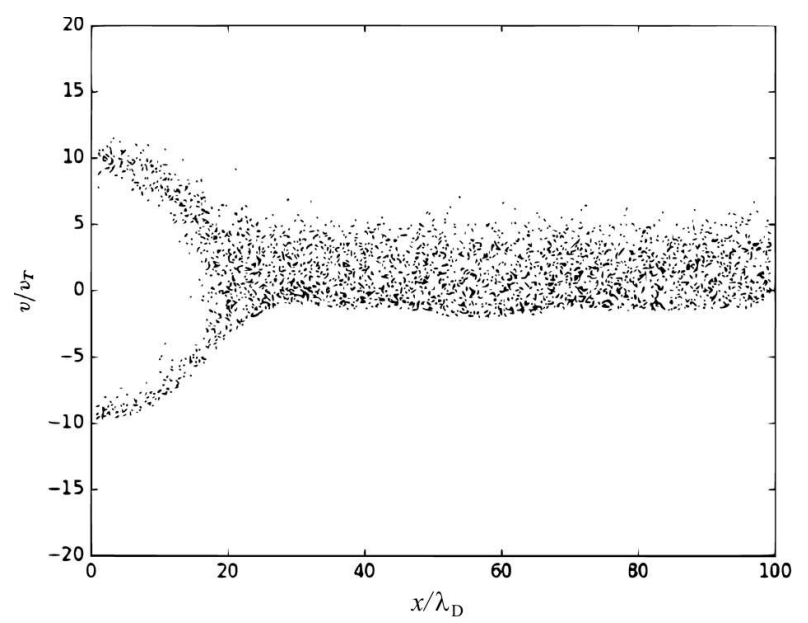

Fig. 2. The phase portrait of the double layer in plasma

plasma with a temperature $\mathrm{kT}$ and an electron concentration $n_{0}$, the ion component of the plasma is frozen and uniformly distributed. A continuous electron beam was injected into the plasma from the left at a rate equal to $10 v_{T}$. Within a short time, approximately equal to $30 / \omega_{p}$, a quasistationary mode is established in the phase space of the system with DL reflecting a part of the beam back, as well as small oscillations in the plasma. A typical view of the phase portrait is shown in Fig. 2.

In $[24,25]$, it was shown that when an electron beam from Io enters Jupiter's ionosphere, a beamplasma instability (BPI) develops. The electron distribution function becomes wider due to the excited fields, and then these electrons cause the ultraviolet aurora. Since BPI develops locally in the inhomogeneous plasma, it can lead to the formation of DL at a certain height. The properties and stability of the formed intense DL, as well as the dynamics of plasma particles, are described. It is noted that the reflection of electron beams from the Jupiter atmosphere can lead to the formation of a semivortex. The effect of the space charge of a decelerated reflected beam and its collision with particles of the partially ionized plasma lead to a gradual expansion of the beam. Thus, the beam is reflected back with a large radius, which can lead to the vortex formation.

\section{Vortex in a Plane $(r, z)$ \\ in a Collisional Magnetized Plasma with a Reflected Electron Beam}

Consider the feasible vortex dynamics of a reflected electron beam near Jupiter in a plane, one of the axes of which is directed along the magnetic field under the presence of electron collisions.

We assume that the axis $z$ is directed toward the surface of Jupiter. The electric double layer has a finite longitudinal size along $z$, approximately equal to $L_{\mathrm{DL}} \approx \sqrt{2} V_{b} / \omega_{p e}[15-17,19]$. Due to the finite radius of the beam, the DL has a finite transverse dimension. Then the DL has not only a longitudinal electric field $E_{z}$, but also a transverse electric field $E_{r}$.

Although the electrons are magnetized, the beam expands in $E_{r}$ due to collisions and forms an obvious half-vortex in the process of reflection from the DL, under the condition, when the radius of a halfvortex exceeds the radius of the beam, that falls on the layer. We will estimate this condition. Since the crossed configuration of the radial electric $E_{0 r}$ and longitudinal magnetic $H_{0}$ fields is maintained in the vicinity of the DL, the electron beam, due to collisions of electrons with a frequency $\nu_{e}$, expands with a velocity

$V_{r}=-\frac{e \nu_{0 e} E_{0 r}}{m_{e} \omega_{\mathrm{He}}^{2}}$,

where the field $E_{0 r}$ is determined by the space charge of the DL, and $\omega_{\mathrm{He}}=e H_{0} / m_{0 e} c$ is the cyclotron frequency of electrons. For close longitudinal and radial dimensions of the double layer, we have approximately $E_{0 r} \approx E_{0 z}$. We note that the amplitude of the electric potential of the DL is approximately equal to the kinetic energy of the beam, and the length is approximately equal to $L_{\mathrm{DL}} \approx \sqrt{2} V_{b} / \omega_{\text {pe }}[15-17,19]$. Therefore, for the formation of a half-vortex, one can obtain the condition

$\frac{\nu_{e}}{\omega_{\mathrm{He}}^{2}} \frac{\omega_{p e}}{\pi} \approx 1$

So, $\omega_{p e} \equiv\left(4 \pi n_{0 e} e^{2} / m_{e}\right)^{1 / 2}-$ the electron plasma frequency must be greater than $\omega_{\mathrm{He}}$, if $\omega_{\mathrm{He}}$ is greater than $\nu_{e}$.

\section{Vortex in the Plane $(r, \theta)$}

in the Collisionless Approximation

in a Magnetized Plasma with a Reflected Electron Beam

Now, we consider the vortex dynamics of a reflected electron beam near Jupiter in a plane orthogonal to the magnetic field. In the vicinity of DL, there is a crossed configuration of the radial electric $E_{0 r}$ and the longitudinal magnetic field $H_{\mathrm{o}}$. So, vortices can also 
be formed in the plane $(r, \theta)$, because the nonequilibrium state is maintained due to the drift of electrons along the angle $\theta$ with a velocity

$V_{\theta 0}=-\frac{e E_{0 r}}{m_{e} \omega_{\mathrm{He}}}=\left(\frac{\omega_{p e}^{2}}{2 \omega_{\mathrm{He}}}\right)\left(\frac{\Delta n}{n_{0 e}}\right) r \equiv r \omega_{\theta 0}$.

$\Delta n \equiv n_{0 e}-q_{i} n_{0 i} / e$.

where $q_{i}, n_{0 i}$ - ion charge and density.

Consider a vorticity $\alpha$ - an electron vortex characteristic

$\alpha \equiv \mathbf{e}_{z} \operatorname{rot} \mathbf{V}=\frac{1}{r} \partial_{r} r V_{\theta}-\frac{1}{r} \partial_{\theta} V_{r}$

The physical sense of $\alpha$ becomes obvious, if we introduce the angular velocity of the electron rotation in a vortex $\Omega \equiv V_{\theta} / r$, then

$\alpha=2 \Omega+r \partial_{r} \Omega-\frac{1}{r} \partial_{\theta} V_{r}$.

If $\Omega \neq \Omega(r)$ and $V_{r}=0$, then the vorticity is equal to the double angular velocity of the electron rotation $\alpha=2 \Omega$.

\section{Equations Describing the Excitation of Nonlinear Vortex Perturbations}

Let us obtain the equation that describes the excitation and properties of vortex perturbations. In [26], the derivation of the equation for the vorticity begins with the momentum equation (2) for a viscous Newtonian conducting fluid, in which there is no electric field. We use the hydrodynamic equations for electrons at times shorter than the capture with regard for electron collisions

$$
\begin{aligned}
& \frac{\partial \mathbf{V}}{\partial t}+\nu_{e} \mathbf{V}+(\mathbf{V} \boldsymbol{\nabla}) \mathbf{V}= \\
& =\left(\frac{e}{m_{e}}\right) \boldsymbol{\nabla} \varphi+\left[\boldsymbol{\omega}_{\mathrm{He}}, \mathbf{V}\right]-\left(\frac{V_{\mathrm{th}}^{2}}{n_{e}}\right) \boldsymbol{\nabla} n_{e}, \\
& \frac{\partial n_{e}}{\partial t}+\boldsymbol{\nabla}\left(n_{e} \boldsymbol{V}\right)=0
\end{aligned}
$$

and the Poisson equation for the electric potential $\varphi$

$\Delta \varphi=4 \pi\left(e n_{e}-q_{i} n_{i}\right)$,

where $\mathbf{V}$ and $n_{e}$ are the electron velocity and density, respectively, $V_{\mathrm{th}}$ - thermal electron velocity, $\mathbf{V}_{i}$, $n_{i}$, and $q_{i}$ - ion velocity, density, and charge. As we will see below, the dimensions of vortex disturbances are much larger than the Debye electron radius $r_{d e} \equiv V_{\mathrm{th}} / \omega_{p e}$. Therefore, we can neglect the last term in (5).

ISSN 2071-0194. Ukr. J. Phys. 2021. Vol. 66, No. 4
We obtain a unified nonlinear equation describing the vortex dynamics of electrons. For this, we use rot for $(5)$, i.e. we act vectorially by the operator $\nabla \times$ on (5). Then we get

$$
\frac{\partial \boldsymbol{\alpha}}{\partial t}+\nu_{e} \boldsymbol{\alpha}+[\boldsymbol{\nabla} \times(\mathbf{V} \boldsymbol{\nabla}) \mathbf{V}]=\left[\boldsymbol{\nabla} \times\left[\boldsymbol{\omega}_{\mathrm{He}} \times \boldsymbol{V}\right]\right]
$$

Here, $\boldsymbol{\alpha}=[\boldsymbol{\nabla} \times \mathbf{V}]$. To transform the last equation we use the expression

$\left[\boldsymbol{\nabla} \times\left[\boldsymbol{\omega}_{\mathrm{He}} \times \mathbf{V}\right]\right]=(\boldsymbol{\nabla} \mathbf{V}) \boldsymbol{\omega}_{\mathrm{He}}-\left(\boldsymbol{\nabla} \boldsymbol{\omega}_{\mathrm{He}}\right) \mathbf{V}=$

$=\boldsymbol{\omega}_{\mathrm{He}}(\boldsymbol{\nabla} \mathbf{V})+(\mathbf{V} \boldsymbol{\nabla}) \boldsymbol{\omega}_{\mathrm{He}}-\left(\boldsymbol{\omega}_{\mathrm{He}} \boldsymbol{\nabla}\right) \mathbf{V}$

at $\nabla \boldsymbol{\omega}_{\mathrm{He}}=0$ and

$[\mathbf{V} \times[\boldsymbol{\nabla} \times \mathbf{V}]]=[\mathbf{V} \times \boldsymbol{\alpha}]=1 / 2 \boldsymbol{\nabla} V^{2}-(\mathbf{V} \boldsymbol{\nabla}) \mathbf{V}$.

From (9), we get the expression

$[\boldsymbol{\nabla} \times(\mathbf{V} \boldsymbol{\nabla}) \mathbf{V}]=-[\boldsymbol{\nabla} \times[\mathbf{V} \times \boldsymbol{\alpha}]]=$

$=-(\boldsymbol{\nabla} \boldsymbol{\alpha}) \mathbf{V}+(\boldsymbol{\nabla} \mathbf{V}) \boldsymbol{\alpha}=$

$=(\mathbf{V} \boldsymbol{\nabla}) \boldsymbol{\alpha}+\boldsymbol{\alpha}(\boldsymbol{\nabla} \mathbf{V})-(\boldsymbol{\alpha} \boldsymbol{\nabla}) \mathbf{V}-\mathbf{V}(\boldsymbol{\nabla} \boldsymbol{\alpha})$.

From (7), (8), (10), and $\boldsymbol{\nabla} \boldsymbol{\alpha}=0$, we find

$\partial_{t} \boldsymbol{\alpha}+\nu_{e} \boldsymbol{\alpha}+(\mathbf{V} \boldsymbol{\nabla}) \boldsymbol{\alpha}+\boldsymbol{\alpha}(\boldsymbol{\nabla} \mathbf{V})-(\boldsymbol{\alpha} \boldsymbol{\nabla}) V=$

$=\boldsymbol{\omega}_{\mathrm{He}}(\boldsymbol{\nabla} \mathbf{V})+(\mathbf{V} \boldsymbol{\nabla}) \boldsymbol{\omega}_{\mathrm{He}}-\left(\boldsymbol{\omega}_{\mathrm{He}} \boldsymbol{\nabla}\right) \mathbf{V}$.

Hence, we have

$d_{t}\left(\boldsymbol{\alpha}-\boldsymbol{\omega}_{\mathrm{He}}\right)+\nu_{e} \boldsymbol{\alpha}+\left(\boldsymbol{\alpha}-\boldsymbol{\omega}_{\mathrm{He}}\right)(\boldsymbol{\nabla} \mathbf{V})=$

$=\left(\left(\boldsymbol{\alpha}-\boldsymbol{\omega}_{\mathrm{He}}\right) \boldsymbol{\nabla}\right) \mathbf{V}$,

$d_{t} \equiv \partial_{t}+(\mathbf{V} \boldsymbol{\nabla})$

We transform the third term on the left side of (12) as follows:

$\left(\boldsymbol{\alpha}-\boldsymbol{\omega}_{\mathrm{He}}\right)(\boldsymbol{\nabla V})=-\left(\frac{\boldsymbol{\alpha}-\boldsymbol{\omega}_{\mathrm{He}}}{n_{e}}\right) \times$

$\times\left[\partial_{t}+(\mathbf{V} \boldsymbol{\nabla})\right] n_{e}=-\left(\frac{\boldsymbol{\alpha}-\boldsymbol{\omega}_{\mathrm{He}}}{n_{e}}\right) d_{t} n_{e}$.

From (12) and (13), we find

$d_{t} \mathbf{W}+\nu_{e} \frac{\boldsymbol{\alpha}}{n_{e}}=(\mathbf{W} \boldsymbol{\nabla}) \mathbf{V}$,

where

$\mathbf{W} \equiv \frac{\boldsymbol{\alpha}-\boldsymbol{\omega}_{\mathrm{He}}}{n_{e}}$

Note that, in the case of a collisionless plasma $\left(\nu_{e}=0\right)$, Eq. (14) for the vector $\mathbf{W}$ formally coincides with the Helmholtz equation (see [27,28]) for the vorticity in the case of an incompressible fluid in the 
absence of a magnetic field $\mathbf{H}: d_{t} \boldsymbol{\alpha}=(\boldsymbol{\alpha} \boldsymbol{\nabla}) \mathbf{V}$. Thus, we have obtained a nonlinear vector equation describing the vortex dynamics of electrons.

Let us show that the vortical motion begins, as soon as there appears a perturbation of the electron density. For the transversal electron velocity $\mathbf{V}_{\perp}$ from (5), one can obtain the following equation:

$\mathbf{V}_{\perp}=-\left(\frac{e}{m_{e} \omega_{\mathrm{He}}}\right)\left[\mathbf{e}_{z}, E_{0 r}\right]+\left(\frac{e}{m_{e} \omega_{\mathrm{He}}}\right)\left[\mathbf{e}_{z}, \nabla_{\perp} \phi\right]-$ $-\frac{1}{\omega_{\mathrm{He}}} \partial_{t}\left[\mathbf{e}_{z}, \mathbf{V}_{\perp}\right]-\frac{1}{\omega_{\mathrm{He}}}\left[\mathbf{e}_{z},\left(\mathbf{V}_{\perp} \boldsymbol{\nabla}_{\perp}\right) \mathbf{V}_{\perp}\right]$

$\nabla_{\perp} \varphi \equiv \nabla_{\perp} \phi-E_{0 r}$

where $\phi$ is the electric potential of the vortical perturbation, and $E_{0 r}$ is the radial electrical field.

Taking higher linear terms into account, we derive the following expression from Eqs. (15):

$$
\begin{aligned}
& \mathbf{V}_{\perp} \approx \mathbf{V}_{\theta o}+\left(\frac{e}{m_{\mathrm{e}} \omega_{\mathrm{He}}}\right)\left[\mathbf{e}_{z}, \nabla_{\perp} \varphi\right]+ \\
& +\left(\frac{e}{m_{e} \omega_{\mathrm{He}}^{2}}\right) \partial_{t} \nabla_{\perp} \varphi .
\end{aligned}
$$

From (3) and (16), we obtain the expression for the vorticity:

$$
\alpha \approx\left(\frac{\omega_{p e}^{2}}{\omega_{\mathrm{He}}}\right)\left(\frac{\Delta n}{n_{0 e}}\right)+\frac{e}{m_{e}} \partial_{t} \mathbf{e}_{z}\left[\nabla_{\perp},\left(\frac{1}{\omega_{\mathrm{He}}^{2}}\right) \nabla_{\perp} \varphi\right] .
$$

From (17), we get the expression for the unperturbed value of the vorticity:

$\alpha_{\mathrm{o}} \approx-\frac{2 e E_{0 r}}{m_{e} \omega_{\mathrm{He}}}=\left(\frac{\omega_{p e}^{2}}{\omega_{\mathrm{He}}}\right)\left(\frac{\Delta n}{n_{0 e}}\right)$.

Expression (17) is convenient for a physical interpretation of the connection between the electron density perturbation $\delta n_{e}$ and the vortical motion. To see this, we consider the limiting case and neglect the ion motion. In this case, (17) yields approximately:

$\alpha \approx\left(\frac{\omega_{p e}^{2}}{\omega_{\mathrm{He}}}\right)\left(\frac{\Delta n}{n_{0 e}}\right)+\left(\frac{\omega_{p e}^{2}}{\omega_{\mathrm{He}}}\right)\left(\frac{\delta n}{n_{0 e}}\right)$.

The first term in (19) specifies the electron movement on the closed trajectories in the crossed fields. The second term in (19) shows that the vortical motion begins, as soon as there appears a perturbation of the electron density $\delta n_{e}$. From (19), it also follows that the vorticity has one sign in all beam areas in the case of vortical perturbations of small amplitudes. The opposite sign of the vorticity occurs in some regions of the beam, where the vortex amplitude exceeds a certain value.

\section{Conclusions}

The Juno measurements of the aurora reveal the valuable information about the precipitating particle population, which interacts with the Jovian ionosphere plasma at varying altitudes. More detailed measurements of auroral structures from satellite trails in the infrared and ultraviolet ranges have been obtained in $[29,30]$. The fine structure is observed on the scale of approximately tens of kilometers. It reveals that, in the case of the IFT trail, the emission has an alternating series of spots, reminiscent of vortices which are at small distances from one another and sometimes split into two arcs. The research of the vortex structure formation mechanism can give an understanding of the reasons for these morphologies and of Jovian auroral processes.

In this paper, the vortex dynamics of electron beams is considered in the framework of the IoJupiter interaction. Currents flow from Io along the magnetic IFT and near the poles. These particles impact the ionosphere plasma, and, in the IFT footprint area, the auroras are generated.

The conditions of the formation of vortex perturbations and its properties and dynamics in the crossed configuration of the radial electric and longitudinal magnetic fields are described. The velocity of radial expansion of the electron beam due to electron collisions is taken into account. The nonlinear vector equation is obtained that describes the vortex dynamics of electrons. It is also analyzed how the vortex motion depends on the occurrence of electron density perturbations.

The work was supported by Program of Fundamental Research of Physics and Astronomy Department of the NAS of Ukraine (project No. 0120U101347). The work was performed with the partial support of the Target Complex Program of the NAS of Ukraine on Plasma Physics.

1. T.D. Carr, M.D. Desch, J.K. Alexander. Phenomenology of Magnetospheric Radio Emissions, Physics of the Jovian Magnetosphere. Edited by A.J. Dessler (Cambridge Univ. Press, 1983).

2. N. Krupp et al. Dynamics of the Jovian Magnetosphere, in Jupiter: Planet, Satellites, Magnetosphere. Edited by F. Bagenal (Cambridge Univ. Press, 2004) [ISBN: 0-52181808-7].

3. J.T. Clarke et al. Ultraviolet emissions from the magnetic footprints of Io, Ganymede and Europa on Jupiter. Nature 415 (6875), 997 (2002).

ISSN 2071-0194. Ukr. J. Phys. 2021. Vol. 66, No. 4 
4. J.E.P. Connerney et al. Jupiter's magnetosphere and aurorae observed by the Juno spacecraft during its first polar orbits. Science 356, 826 (2017).

5. B.H. Mauk, D.K. Haggerty et al. Discrete and broadband electron acceleration in Jupiter's powerful aurora. Nature 549, 66 (2017).

6. W.R. Dunn, G. Branduardi-Raymont et al. The independent pulsations of Jupiter's northern and southern X-ray auroras. Nature. Astronomy 1, 758 (2017).

7. D.J. McComas, N. Allegrini et al. The Jovian auroral distributions experiment (JADE) on the Juno mission to Jupiter. Space Sci. Rev. 213, 547 (2017).

8. B.H. Mauk et al. Juno observation of energetic charged particles over Jupiter's polar regions: Analysis of monodirectional and bidirectional electron beams. Geophys. Res. Lett. 44, 4410 (2017).

9. W.S. Kurth, M. Imai et al. A new view of Jupiter's auroral radio spectrum. Geophys. Res. Lett. 44, 7114 (2017).

10. P.I. Fomin, A.P. Fomina. Dicke superradiance on Landau levels. Probl. of atomic sci. and techn. 1, 45 (2001).

11. V.M. Mal'nev, A.P. Fomina, P.I. Fomin. Polarization phase transition to the superradiance regime of the inverted system of electrons on high Landau levels. Ukr. J. Phys. 47, 1001 (2002).

12. P.I. Fomin, A.P. Fomina, V.N. Mal'nev. Superradiation of magnetized electrons and the power of decameter radiation of the Jupiter-Io system. Ukr. J. Phys. 49, 3 (2004).

13. O.P. Novak, A.P. Fomina, R.I. Kholodov. Account of the longitudinal temperature in cyclotron superradiance. Probl. of Atomic Sci. and Techn. 85, 69 (2013).

14. O. Novak, R. Kholodov, A. Fomina. Role of double layers in the formation of conditions for a polarization phase transition to the superradiance state in the Io flux tube. Ukr. J. Phys. 63, 740 (2018).

15. V.I. Maslov. The double layer formed by a nonrelativistic electron beam in the one-dimensional plasma. Ukr. J. Phys. 33, 1342 (1988).

16. V.I. Maslov. Electron beam reflection from the plasma due to double layer formation. In: Proc. of 4 th Int. Workshop on Nonlinear and Turbulent Processes in Physics (Singapore, 1990), p. 898.

17. V.I. Maslov. Properties and evolution of nonstationary double layers in nonequilibrium plasma. In: Proc. of 4 th Symposium on Double Layers and Other Nonlinear Structures in Plasma (Innsbruck, 1992), p. 82

18. V.I. Maslov. Double layer formed by a relativistic electron beam. Sov. J. of Plasma Phys. 18, 676 (1992).

19. V.I. Maslov, V.V. Oraevsky, Yu.Ya. Ruzhin. Ion acceleration in collective fields at electron beam injection from spacecraft in experiment "APEX". Phys. Scr. 57, 453 (1998).

20. V. Lapshin, V. Maslov, V. Stomin. Analytical description of T. Sato's mechanism of transformation of ion-acoustic double layer into strong Buneman's one in cosmic and laboratory nonequilibrium plasmas. J. Plasma Fusion Res. Ser. 4, 564 (2001).
21. Ie.V. Borgun, N.A. Azarenkov, A. Hassanein, A.F. Tseluyko, V.I. Maslov, D.L. Ryabchikov. Double layer influence on dynamic of the EUV radiation from plasma of the high-current pulse diode in the tin vapour. Phys. Lett. A 377 (3-4), 307 (2013).

22. M.A. Raadu. The physics of double layers and their role in astrophysics. Phys. Rep. 178, 25 (1989).

23. R.E. Ergun, Y.J. Su, L. Andersson et al. Direct observation of localized parallel electric fields in a space plasma. Phys. Rev. Lett. 87, 045003 (2001).

24. V.I. Maslov, I.P. Levchuk, S. Nikonova, I.N. Onishchenko. Occurrence of accelerating field, formation and dynamics of relativistic electron beam near Jupiter. East Eur. J. Phys. 5, 78 (2018).

25. V.I. Maslov, A.P. Fomina, R.I. Kholodov, I.P. Levchuk, S. Nikonova, O.P. Novak, I.N. Onishchenko. Accelerating field excitation, occurrence and evolution of electron beam near Jupiter. Probl. of Atomic Sci. and Techn. 4, 106 (2018).

26. P.J. Hendricks. Vorticity transport by electromagnetic forces. NUWC-NPT Techn. Report 10, 712 (1998).

27. H. Helmholtz. Uber integralle der hydrodynamischen Gleichungen, welche den Wirbewegungen entsprechen. Crelle J. 55, 25 (1858).

28. W. Thomson. On vortex motion. Trans. Roy. Soc. Edinburgh 25, 217, (1869).

29. C. Paranicas, B. Mauk et al. Intervals of intense energetic electron beams over Jupiter's poles. J. of Geoph. R.: Space Physics 123 (A10), 1989 (2018).

30. A. Mura, A. Adriani, J.E.P. Connerney et al. Juno observations of spot structures and a split tail in Io-induced aurorae on Jupiter. Science 361 (6404), 774 (2018).

Received 27.07.20

В.І. Маслов, О.К. Черемних, А.П. Фоміна,

Р.І. Холодов, О.П. Новак, Р.Т. Овсянніков

ВИХРОВІ СТРУКТУРИ

I ДИНАМІКА ЕЛЕКТРОННОГО ПУЧКА В ЗАМАГНІЧЕНІЙ ПЛАЗМІ

В даній роботі досліджено задачу про формування вихрових структур при відбитті пучка електронів від подвійного шару іоносфери Юпітера та вплив цих структур на виникнення щільних висхідних електронних пучків, прискорених потенціалом подвійного шару вздовж потокової трубки Iо, для яких стає можливий фазовий перехід в режим циклотронного надвипромінення. Розглянуто умови формування вихрових збурень. Знайдено нелінійне рівняння, яке описує вихрову динаміку електронів, та вивчено його наслідки.

Ключов $i$ слова: динаміка електронного пучка, подвійний електричний шар, механізм відбиття електронів, іоносфера Іо, плазма, вихори. 